\title{
MicroRNAs as biomarkers in molecular diagnosis of refractory epilepsy
}

\author{
Xiaofeng Wang ${ }^{1,2,4+}$, Hang Yin ${ }^{3 \dagger}$, Alyson M. Rich ${ }^{5,6,7}$, Yuqiang Sun ${ }^{2}$, Zeshi Tan ${ }^{2}$, Xiaodong Luo ${ }^{2}$, Ningwei Che ${ }^{2}$,
} Minghai $\mathrm{Wei}^{2^{*}}$ and Jian $\mathrm{Yin}^{2,4^{*}}$

\begin{abstract}
MicroRNA (miRNA) is a type of endogenous non-coding RNA that can regulate cell proliferation, differentiation, invasion, apoptosis and several other biological activities by specially inducing gene silencing, and thereby is related to development and disease in life course. In recent years, researchers have found that miRNAs are closely related with refractory epilepsy. MiRNAs can intervene in the modification of mRNAs, the synthesis of proteins and some the connectivity of signal pathways in pathogenesis of epilepsy. Furthermore, some miRNAs in neurons are of great importance in neuronal differentiation. Therefore, miRNA may play a very important role in the occurrence, development and episodes of refractory epilepsy. These discoveries can provide a new direction for the research of pathogenesis, diagnostic methods and therapeutic approach of refractory epilepsy. Although research about miRNA and intractable epilepsy has progressed, more remains to be done before miRNA can be used in clinical diagnosis and treatment strategies. This paper focuses on the research progress of molecular diagnosis about miRNA in intractable epilepsy.
\end{abstract}

Keywords: MicroRNA, Refractory epilepsy, Biomarker, Diagnosis

Abbreviations: miRNA, MicroRNA; HS, Hippocampal sclerosis; DRE, Drug-resistant epilepsy; ILAE, The international league against epilepsy; EEG, Electroencephalography; SE, Status epileptics; mTLE, Medial temporal lobe epilepsy; CREB, CAMP-response element binding protein

\section{Background}

Epilepsy is a chronic neurologic disorder characterized by recurring abnormal electricity activity in brain, which is estimated to afflict about 65 million individuals worldwide [1]. Approximately $10 \%$ of the publics have at least one seizure during their lifetime and among them about $30 \%$ will develop epilepsy, suffering substantial deleterious influence on their health due to long-term use of drugs [2]. Nowadays it lacks a complete understanding of the epileptogenic process but it is thought to involve neuronal death or dysfunction, neurogenesis and others [2]. Many researchs now support miRNAs as a critical layer of gene expression regulation in epilepsy, which provides new putative targets for the control of seizures, seizure-

\footnotetext{
*Correspondence: weimingh@sina.cn; dyeyDr_yin@163.com

${ }^{\dagger}$ Equal contributors

2Department of Neurosurgery, The Second Affiliated Hospital of Dalian

Medical University, 467 Zhongshan Road, Shahekou, Dalian, Liaoning 116044,

People's Republic of China

Full list of author information is available at the end of the article
}

induced damage and as potential biomarkers of epileptogenesis. miRNA is detected in many biofluids, including serum and CSF and it appears to be very stable $[3,4]$.

Current strategies to fight epilepsy focus on arresting epileptogenesis induced by brain trauma and other diseases through treatment of anti-epileptic drugs [3]. Substantial research has focused on the involvement of microRNAs in epilepsy, including screening the aberrant expression of microRNAs and searching their target genes. The isolation of miRNAs' target genes would enable the development of a better understanding of the etiology, and the establishment of diagnostic biomarkers, and the identification of novel miRNA-based therapeutics.

\section{Drug-resistant epilepsy: definition and prognosis}

Globally, the estimated proportion of the general population with active epilepsy is about $0.5-1 \%$ and $20-30 \%$ of those patients will develop a drug-resistant epilepsy (DRE) $[4,5]$. Clinical trials demonstrated that cerebrovascular diseases, encephalitis and cortical dysplasia 
function as main causes of DRE. Diseases including West syndrome, Lennox-Gastaut syndrome and tuberous sclerosis could assist in identifying DRE [6]. Moreover, temporal lobe epilepsy (TLE) represents the most common syndrome in adults and often develops pharmacoresistence [7]. TLE frequently develops into DRE as a result of hippocampal sclerosis (HS) [8-10], inflammation [11], neurocysticercosis [12], brain insult [13], or malformations of cortical development [14, 15]. However, a consensus definition and prognosis for DRE remained elusive [16, 17]. In 2010, the International League against epilepsy (ILAE) addressed the need for a definition, declaring that"Drug resistant epilepsy may be defined as failure of adequate trials of two tolerated and appropriately chosen and used AED schedules (whether as monotherapies or in combination) to achieve sustained seizure freedom" [18]. This proposal provided a precise definition of DRE but, left forming an efficacious prognosis method for DRE unexplored to this day. Past early-identification of DRE is mainly based on clinical manifestations like the number of seizures [19]. Other than applying the well-established electroencephalography (EEG) monitoring, other imaging testsing and clinical symptoms and signs watching, DRE urgently requires a set of molecular biomarkers to clarify the early prognosis, wherein microRNAs play an important role [20-22].

\section{MicroRNA: biogenesis and regulatory mechanisms} miRNA is a non-coding single-stranded RNA molecule with length of about 20-24 nucleotides and has highly conservatism, tissue specificity and temporality. It does not have open reading frame and mainly regulates expression of gene after transcription [23]. Most miRNA genes are present in the genome as the form of single copy, multiple copies or a gene cluster. Each miRNA can regulate one or more target genes, while several miRNAs can regulate the same target gene. miRNA regulates the expression level of proteins in cells through this complicated network [24]. Essentially, miRNA is a highly conserved non-coding single-stranded endogenous RNA molecule that plays an important role in the negative regulation of the target gene expression after transcription [25].

\section{MicroRNAs in animal experiments of DRE prognosis}

In animal experiments about DRE, researchers mainly focused on temporal lobe epilepsy and hippocampal sclerosis by artificially modeling mice or rats. Researchers further compartmentalized subgroups by distinguishing different time phases of seizures, different locations and different injury statuses of the brain.
MicroRNAs profiling in different time phases after SE

The detection of abnormal expression of miRNAs in tissue is usually obtained during status epilepticus (SE) and after induction of SE at 1 day, 1 week or over 2 months. Distinct expression levels of miRNAs have been discovered at all time points. Thus, researchers considered the alteration of miRNA levels in interictal periods and chronic stage to have great diagnostic significance. To date, over 350 different miRNAs are postulated to closely correlate with epileptogenesis and epilepsy development while only up to $20 \%$ of these miRNAs have been identified in brain tissues of epilepsy animal models [26]. Researchers suppose that such heterogeneity may be the result of the influence exerted by epilepsyinduced brain tissue damage to miRNA detection, thus choosing to conduct miRNA profiling in brain tissues extracted at least 2 months (chronic stage) after epileptic seizures. Four animal-based studies utilizing gene chips to identify miRNA expression, respectively conducted by Song et al. [26] Hu et al. [27] Bot et al. [28] and Gorter et al. [29], together reported about 25 differentially expressed miRNAs. Those mentioned in more than one of the studies include 14 up-regulated miRNAs and 9 down-regulated miRNAs. Up-regulated miRNAs includes miR-21, miR-23a, miR-23b, miR-24, miR-27a, miR-27b, miR-34a, miR-126, miR-132, miR-140, miR146a, miR-152, miR-210 and miR-212 while downregulated miRNAs includes miR-33, miR-138, miR-139, miR-187, miR-190, miR-218a, miR-301a, miR-551b and miR-935. Among the up-regulated miRNAs only three were mentioned in three human experiments, which are miR-21, miR-132 and miR-146a [30-32]. Of note, miR134 is also an important microRNA that is up-regulated during epilepsy, which is only profiled in one human experiment of Jimenez-Manteos et al. [23].

\section{MicroRNAs profiling in different damage status of brain}

Through distinguishing differential damage statuses (including intercerebral hemorrhagic injury $(\mathrm{H})$, ischemic injury (I) and kainate seizures (K)) of rat brains after epileptic seizures in $24 \mathrm{~h}$, Liu et al. [33] identified $91 \mathrm{miR}$ NAs in brain (I+:24, $\mathrm{H}+: 17, \mathrm{~K}+: 13$; I-:13, $\mathrm{H}-: 12, \mathrm{~K}-: 18)$ and 147 miRNAs in blood $(\mathrm{I}+: 10 \mathrm{H}+: 21 \mathrm{~K}+: 21 \mathrm{I}-: 65 \mathrm{H}-$ :20 K-:10) that exhibits abnormal expressions (fold change $>1.5$ ) in three damage statuses. Among them, miRNAs that show aberrant expression in mice brain tissue of all three damage statuses includes up-regulated miR-542-3p and down-regulated miR-122, miR-155, miR-362-3p and miR-450-5p. In blood, five up-regulated miRNAs were clearly documented miR-96, miR-152, miR-298, miR-333 and miR-505. Seven down-regulated miRNAs were also found in the blood: being miR-125a5p, miR-130b, miR-142-3p, miR-330, miR-342-5p, miR347 and miR-685 miRNAs that are both regulated in 
brain and blood include miR-298, miR-503, miR-672, miR-10a, miR-182 and miR-200b (SI \& I+); miR-155, miR-362-3p, miR-223 and miR-210 (SI \& I-); miR-148b, miR-503, miR-298, miR-423-5p, miR-10a, miR-345-5p and miR-674 (SH \& LB(H)+); miR-10b, miR-203, miR362-3p, miR-155, miR-20b-3p and miR-200a (SH \& LB(H)-); miR-298 (K+); miR-155, miR-29c, miR-34b-3p, miR-98, miR-122, miR-203 and miR-450a (K-). Since considerable amounts of miRNAs have been successfully identified, future studies should focus more on the clinical significance of particular miRNAs used as biomarkers or potential therapeutic targets.

\section{MicroRNAs profiling in different area of brain after SE}

Jan et al. [34] have analyzed the miRNA expression in three brain regions - hippocampal CA1, hippocampal dentate gyrus and parahippocampal cortex-correlated with TLE in rats and obtained tissue after electrically-induced SE at 1 day, 1 week and 3-4 months. Results revealed that 18, 16 and 7 miRNAs were identified respectively in the hippocampal CA1 in the aforementioned three time phases. Among them, miR-21 exhibits high expression in both tissue obtained in 1 day and 1 week after SE induction and miR-143, miR-23a, miR-27a showed upregulation in tissue obtained after 1 week and 3-4 months. In the hippocampal dentate gyrus, there were 20 , 15 and 37 miRNAs respectively detected in the three time phases. Three miRNAs - miR-132, miR-21, miR-212 - exhibited up-regulation in all three time phases while miR142-5p was up-regulated in 1 days and 1 weeks after SE. miR-146a, miR-212, miR-23a were up-regulated in 1 day and 3-4 months after SE and miR-223 showed upregulation in 1 day and 3-4 months after SE. In the parahippocampal cortex, 31, 37 and 22 miRNAs respectively were profiled in three time phases. miR-21 and miR-223 displayed up-regulation in all three time phases; $12 \mathrm{miR}$ NAs (miR-142-5p, miR-294, miR-32, miR-351, miR-3596c, miR-466b-1, miR-466b-2, miR-466c, miR-466d, miR-675, miR-758 and miR-883)were up-regulated in 1 day and 1 week after SE samples,; and 6 miRNAs (miR-193, miR199a-3p, miR-199a-5p, miR-23a, miR-27a, miR-34a) presented up-regulation in both 1 week and 3-4 months. However, extremely few miRNAs were identified in the hippocampal CA1 and dentate gyrus and not a single miRNA was identified in all three time phases. In the parahippocampal cortex, respectively 68, 102 and $7 \mathrm{miR}$ NAs were identified in three time phases while only miR137 was down-regulated in all three time phases. Currently no research has focused on the relationship between miR-137 and intractable epilepsy. Aside from brain tissue, Jan et al. also profiled miRNAs in plasm and 3 miRNAs were detected, being miR-142-5p detected in 1 day after SE, miR-21-5p in 1 week after SE and miR-146-5p in
3-4 months after SE. No miRNA was detected in two or three time phases.

Although such substantial miRNAs were identified in tissue and plasm in different time phases, areas and damage statuses, few miRNAs show essential clinical significance. Blondal et al. [35] assessed 119 miRNAs that are most commonly found in normal plasm, wherein 9 miRNAs are overlapped with those identified by Jan et al. in brain tissue and 8 miRNAs plasma ( 5 tissue +3 plasm) are overlapped with those detected by Liu et al. in brain tissue and plasm. These respectively are miR-21, miR-142-5p, miR-146a, miR-23a, miR-223, miR-32, miR199a-3p, miR-199a-5p, miR-27a (Jan et al. Tissue overlapped), miR-122, miR-152, miR-505, miR-125a-5p, miR142-3p (Liu et al. Tissue overlapped), miR-20b, miR-29c, miR-122 (Liu et al. Plasm overlapped) The scarcity of these findings indicates that these miRNAs may have litthe relation with epileptogenesis. However, microRNAs such as miR-146a are still validated to be highly implicated in epileptogenesis. Thus, whether those 17 microRNAs are involved in epileptogenesis or not requires further discussion.

Based on this research, approximately 26 miRNAs exert a direct or indirect influence in animal experiments of epilepsy diagnosis, being miR-542-3p, miR-362-3p, miR-4505p, miR-96, miR-143, miR-23a, miR-155, miR-152, miR298, miR-333, miR-330, miR-342-5p, miR-347, miR-34, miR-294, miR-351, miR-466 family (b-1/b-2/c), miR-675, miR-758, miR-883, miR-193, miR-134, miR-132, miR146a. Other microRNAs may also play a role in epilepsy, but this remains to be explored.

\section{MicroRNAs in human experiment of DRE diagnosis}

Two studies published in 2012 on miRNA profiles in TLE revealed a considerable amount of dysregulated miRNAs in human diseased tissue. Kan et al. [36] focused on medial temporal lobe epilepsy (mTLE), a subtype of TLE caused by hippocampal sclerosis [37]. They performed genome-wide miRNA profiling in 20 human hippocampal tissue samples, including both sclerotic and non-sclerotic. The study also identified 51 strongly regulated miRNAs, wherein 11 miRNAs play essential roles. Of the 11 miRNAs, 7 were up-regulated- miR-190, miR-20a, miR-32, miR-374a, miR-26b, miR-193a-3p, miR-92b and 4 were down-regulated - miR-184, miR-138, miR-221, miR222. Kan et al. found that miR-92b, miR-637, and miR-665 showed inconsistent localization between being prominent in the cytoplasm of neurons in autopsy controls and being in the nucleus of both neurons and astrocytes in mTLE samples. However, miR-138 expression was limited to the cytoplasm in mTLE patients. These findings together suggest that the localization of a specific miRNA may have significant clinical applications [38]. Consequently, detecting 
the nuclear expression of miR-92a, miR-637 and miR-665 may be a useful method for epilepsy diagnosis. Furthermore, 16 miRNAs involved in epileptogenesis were identified by summarizing the meaningful results from many studies, including miRNAs modulating neuronal maturation (miR-137 [39]), neural cell proliferation in cortex (miR-9 [40], miR-124 [41, 42], miR-137 [40, 43], miR-184 $[40,44,45]$ and let-7b $[40,43,46])$, and neural differentiation (miR-153 [47], miR-324 [47], miR-181a [47], miR-34a $[27,48])$. Other miRNAs are reported to be critical in neuronal migration (miR-9 [49], miR-134 [23], miR-137 [50]), neuronal differentiation (miR-125b [51] miR-137 [40]) and neural inflammation (miR-146a [52]).

Research on epilepsy-related microRNAs in human experiments typically progress slowly because factors key factors such as drug treatment influence miRNA profiling results. No clear result has been established on the relationship between microRNA and intractable epilepsy in human experiments, leaving a huge development space for applying microRNAs to potential biomarkers and therapeutic targets in refractory epilepsy. In this regard, it is a promising research direction to profile miRNA expression in both diseased brain tissue and corresponding plasm samples, and then compare fold changes of miRNA expressions to those in control groups.

\section{MicroRNAs expression regulation in epilepsy}

The mechanism of down-regulation of a series of miRNA in TLE patients is well understood. McKiernan et al. [45] profiled miRNA expression in sclerotic hippocampi from TLE patients and found a significant down-regulation of 37 miRNAs out of the total 380 human miRNAs. In order to identify the cause of these miRNAs' collapse, McKiernan et al. measured the transcript level of 4 pri-miRNAs and found no reduction of their levels, but a prominent decrease in Dicer level was identified. Dicer is an RNase III essential to the cleavage of pre-miRNA to mature miRNA. The deletion of Dicer in the neural system typically leads to neuronal dysfunction, neurodegeneration, macular degeneration, epilepsy seizure and cognitive deficits [53-57]. The loss of Dicer appears specific to TLE patients with HS and cause a failure of miRNA biogenesis, which further gives rise to degenerative changes in cortex and hippocampal morphology that have been observed in human epilepsy [54]. Aside from Dicer, other components of miRNA biogenesis may also play a role in miRNA expression regulation and may provide potential explanation for specific miRNA up-regulation. McKiernan et al. [45] also noticed that Ago2 levels were higher in non-sclerotic TLE patients. This indicates that epileptic seizures may up-regulate AGO2, by which miR-451, an up-regulated miRNA in TLE tissue, could be processed independently of Dicer [58, 59].
There are two other established miRNA regulators of note in the neural system and epilepsy: cAMP-response element binding protein (CREB) and transcription factors. CREB that regulates miR-132, contributing to the development of synaptic network plasticity. Transcription factors like myocyte enhancing factor 2 (Mef2) up-regulate the miR379-410 cluster, wherein miR-134 exhibits the essential role of promoting dendritic outgrowth of hippocampal neurons by inhibiting translation of translational repressor Pumilio2 mRNA [60, 61].

\section{Pathogenetic mechanisms of miRNA-mediated epileptogenesis}

Although miRNA is predicted to regulate the expression of hundreds of proteins involved in wide-ranging biological processes, the processes regulated by miRNA may be restricted in epilepsy [23]. Inflammation and neuron morphology modulation may be two of the most prominent regulatory roles of miRNA in epileptogenesis [22]. Similar to miRNA in tumorigenesis, miRNAs exhibit a dual role in epileptogenesis- neuroprotection or induce neural cell death. A large number of studies have shown that the occurrence of epilepsy with nerve cells apoptosis, Synaptic connections reconstruction, glial fibers cell hyperplasia, abnormal pathways is closely related to the formation and inflammatory response [20, 21]. Repeated seizures can result in hippocampus neuron apoptosis in brain, the abnormal formation excitatory synapses loop, and ultimately improve the intractable temporal lobe epilepsy [23, 24]. Thus, miRNAs may be through regulating nerve cells apoptosis, synaptic contact reconstruction, glial fiber cell hyperplasia, inflammation involved in the occurrence of epilepsy development.

\section{Conclusions and expectation}

In the study of human disease, involves only less than 100 kinds of micrornas associated with refractory epilepsy, and collect more meaningful experimental results including up-regulation of miRNAs : miR-146a,-132,-9,99a,-27a,-203,-135a, -4521 [62]; the down-regulation of miRNAs:miR-30a/b,-138,-324,-330,-187,-194-5p,-301a3p,-30b-5p,-342-5p, -4446-3p [63].

Although miRNA research remains in its early stages, it certainly could have great applications in the molecular diagnosis of intractable epilepsy. As research into this area progresses, the miRNA with specificity and sensitivity will likely be found among numerous miRNAs related to epilepsy [64]. In time, this will lead to the development of effective strategies for the early screening, clinical diagnosis, treament and preventation of refractory epilepsy. The research of miRNA involved in epilepsy has great application prospect and huge potential in diagnosis and treatment of intractable epilepsy on the basis of the molecular biology. 


\section{Acknowledgements}

The authors have no personal financial or institutional interest in any of the drugs, materials, or devices described in this article. We gratefully acknowledge the hard work of our team in Epileptic Center of Liaoning, The Second Affiliated Hospital of Dalian Medical University. Without their help, it would be hard to finish this work.

\section{Funding}

Not applicable.

\section{Availability of data and materials}

This paper is a review article. Referred literature in this paper has been listed in the references part. The datasets supporting the conclusions of this article is are available online by searching the PubMed.

\section{Authors' contributions}

All authors read and approved the final manuscript.

\section{Competing interests}

The authors declare that they have no competing interests.

\section{Consent for publication}

Not applicable.

\section{Ethics approval and consent to participate}

Not applicable.

\begin{abstract}
Author details
'Department of Neurosurgery, Weinan Central Hospital, Weinan, Shaanxi 714000, People's Republic of China. ${ }^{2}$ Department of Neurosurgery, The Second Affiliated Hospital of Dalian Medical University, 467 Zhongshan Road, Shahekou, Dalian, Liaoning 116044, People's Republic of China. ${ }^{3}$ Dalian No. 24 high school, Dalian, Liaoning 116044, People's Republic of China. ${ }^{4}$ Department of Neurosurgery, Epileptic Center of Liaoning, The Second Affiliated Hospital of Dalian Medical University, 467 Zhongshan Road, Shahekou, Dalian, Liaoning 116044, People's Republic of China. ${ }^{5}$ Department of Psychiatry, Yale University School of Medicine, 300 George St., New Haven, CT 06511, USA. ${ }^{6}$ Abraham Ribicoff Research Facilities, Connecticut Mental Health Center, 34 Park St., New Haven, CT 06519, USA. ${ }^{7}$ College of Literature, Science, and the Arts, University of Michigan, Ann Arbor, MI 48109, USA.
\end{abstract}

Received: 1 June 2016 Accepted: 23 August 2016

Published online: 18 October 2016

\section{References}

1. Moshe SL, Perucca E, Ryvlin P, et al. Epilepsy: new advances. Lancet. 2015:385(9971):884-98.

2. Devinsky O. Patients with refractory seizures. N Engl J Med. 1999;340(20):1565-70.

3. Annamaria V. Before epilepsy unfolds: finding the epileptogenesis switch. Nat Med. 2012;18:1626-7.

4. Berg AT. Understanding the delay before epilepsy surgery: who develops intractable focal epilepsy and when? CNS Spectr. 2004;9(2):136-44.

5. Shneker BF, Fountain NB. Epilepsy. Dis Mon. 2003;49(7):426-78.

6. Chang BS, Lowenstein DH. Epilepsy. N Engl J Med. 2003;349(13):1257-66.

7. Tatum 4th WO. Mesial temporal lobe epilepsy. J Clin Neurophysiol. 2012;29(5):365-65.

8. Thom M. Hippocampal sclerosis: progress since Sommer. Brain Pathol. 2009;19:565-72

9. Thom M. Reivew: Hippocampal sclerosis in epilepsy: a neuropathology review. Neuropathol Appl Neurobiol. 2012;40(5):520-43.

10. Cendes F, Sakamoto AC, Spreafico R, et al. Epilepsy associated with hippocampal sclerosis. Acta Neuropathol. 2014;128(1):21-37.

11. Dupuis N, Auvin S. Inflammation and epilepsy in the developing brain: clinical and experimental evidence. CNS Neurosci Ther. 2015;21(2):141-51.

12. Nash TE, Del Brutto OH, Butman JA, et al. Calcific neurocysticercosis and epileptogenesis. Neurology. 2004;62(11):1934-8.

13. Herman ST. Epilepsy after brain insult: targeting epileptogenesis. Neurology. 2002;59(9 Suppl 5):S21-6.

14. Guerrini R, Sicca F, Parmeggiani L. Epilepsy and malformations of the cerebral cortex. Epileptic Disord. 2003;5 Suppl 2:S9-\$26.
15. Sutula TP. A glimpse into abnormal cortical development and epileptogenesis at epilepsy surgery. Neurology. 1998;50(1):8-10.

16. Perucca E. Pharmacoresistance in epilepsy. How should it be defined? CNS Drugs. 1998:10:171-9.

17. Berg AT, Vickreg BG, Testa FM, et al. How long does it take epilepsy to become intractable? A prospective investigation. Ann Neurol. 2006;60:73-9.

18. Kwan P, Arzimanoglou A, Anne T. B, et al. Definition of drug resistant epilepsy: Consensus proposal by the ad hoc Task Force of the ILAE Commission on Therapeutic Strategies. Epilepsia. 2010;51(6):1069-77.

19. Kwan P, Brodie MJ. Early identification of refractory epilepsy. N Engl J Med. 2000:342:314-9.

20. David C. Henshall. MicroRNA and epilepsy: profiling, functions and potential clinical applications. Curr Opin Neurol. 2014;27(2):199-205.

21. Galanopoulou AS, Moshé SL. In search of epilepsy biomarkers in the immature brain: goals, challenges and strategies. Biomark Med. 2011;5(5):615-28.

22. Pitkäen A, Lukasiuk K. Molecular biomarkers of epileptogenesis. Biomark Med. 2011:5(5):629-33.

23. Jimenez-Mateos EM, Henshall DC. Epilepsy and microRNA. Neuroscience. 2013;238:218-29.

24. Henshall DC. MicroRNAs in the pathophysiology and treatment of status epilepticus. Front Mol Neurosci. 2013;6:37.

25. Enciu AM, Popescu BO, Gheorghisan-Galateanu A. MicroRNAs in brain development and degeneration. Mol Biol Rep. 2012;39(3):2243-52.

26. Song YJ, Tian XB, Zhang $S$, et al. Temporal lobe epilepsy induces differential expression of hippocampal miRNAs including let-7e and miR-23a/b. Brain Res. 2011;1387:134-40.

27. Hu K, Xie YY, Zhang C, et al. MicroRNA expression profile of the hippocampus in a rat model of temporal lobe epilepsy and miR-34atargeted neuroprotection against hippocampal neurone cell apoptosis poststatus epilepticus. BMC Neurosci. 2012;13:1-14.

28. Bot AM, Debski KJ, Lukasiuk K. Alterations in miRNA levels in the dentate gyrus in epileptic rats. PLoS One. 2013;8:e76051.

29. GorterJA IA, White I. Hippocampal subregion-specific microRNA expression during epileptogenesis in experimental temporal lobe epilepsy. Neurobiol Dis. 2013;62:508-20.

30. Aronica E, Fluiter K, lyer A, et al. Expression pattern of miR- 146a, an inflammation-associated microRNA, in experimental and human temporal lobe epilepsy. Eur J Neurosci. 2010;31:1100-7.

31. Omran A, Peng J, Zhang C, et al. Interleukin-1beta and microRNA-146a in an immature rat model and children with mesial temporal lobe epilepsy. Epilepsia. 2012;53:1215-24

32. Ashhab MU, Omran A, Kong $\mathrm{H}$, et al. Expressions of tumor necrosis factor alpha and microRNA-155 in immature rat model of status epilepticus and children with mesial temporal lobe epilepsy. J Mol Neurosci. 2013;51:950-8.

33. Liu DZ, Tian Y, Ander BP, et al. Brain and blood microRNA expression profiling of ischemic stroke, intracerebral hemorrhage, and kainate seizures. J Cereb Blood Flow Metab. 2010;30:92-101.

34. Gorter JA, lyer A, et al. Hippocampal subregion -specific microRNA expression during epileptogenesis in experimental temporal lobe epilepsy. Neurobiol Dis. 2014;62:508-20.

35. Blondal T, et al. Assessing sample and miRNA profile quality in serum and plasma or other biofluids. Methods. 2013;59:S1-6.

36. Kan AA, van Erp S, Derijck AA, de Wit M, Hessel EV, O'Duibhir E, de Jager W, Van Rijen PC, Gosselaar PH, de Graan PN, Pasterkamp RJ. Genome-wide microRNA profiling of human temporal lobe epilepsy identifies modulators of the immune response. Cell Mol Life Sci. 2012;69:3127-45.

37. Landazuri P. Mesial temporal lobe epilepsy: a distinct electroclinical subtype of temporal lobe epilepsy. Neurodiagn J. 2014;54(3):274-88.

38. Wetherington J, Serrano G, Dingledine R. Astrocytes in the epileptic brain. Neuron. 2008:58(2):168-78.

39. Smrt RD, Szulwach KE, Pfeif-fer RL, Li X, Guo W, Pathania M, et al. MicroRNA miR-137 regulates neuronal maturation by targeting ubiquitin lig- ase mind bomb-1. Stem Cells. 2010;28:1060-70.

40. Krichevsky AM, Sonntag KC, Isacson O, Kosik KS. Specific microRNAs modulate embryonic stem cell-derived neurogenesis. Stem Cells. 2006;24:857-64.

41. Makeyev EV, Zhang J, Carrasco MA, Maniatis T. The MicroRNA miR-124 promotes neuronal differentiation by triggering brain-specific alternative pre-mRNA splicing. Mol Cell. 2007:27(3):435-48.

42. Peng J, Omran A, Ashhab MU, Kong H, Gan N, He F, Yin F. Expression patterns of miR-124, miR-134, miR-132, and miR-21 in an immature rat model and children with mesial temporal lobe epilepsy. J Mol Neurosci. 2013;50(2):291-7. 
43. Silber J, Lim DA, Petritsch C, Persson Al, Maunakea AK, Yu M, Vandenberg SR, Ginzinger DG, James CD, Costello JF, Bergers G, Weiss WA, Alvarez-Buylla A, Hodgson JG. miR-124 and miR-137 inhibit proliferation of glioblastoma multiforme cells and induce differentiation of brain tumor stem cells. BMC Med. 2008;6:14.

44. Liu C, Teng ZQ, Santistevan NJ, Szulwach KE, Guo W, Jin P, Zhao X. Epigenetic regulation of miR-184 by MBD1 governs neural stem cell proliferation and differentiation. Cell Stem Cell. 2010;6(5):433-44.

45. McKiernan RC, Jimenez-Mateos EM, Sano T, Bray I, Stallings RL, Simon RP, Henshall DC. Expression profiling the microRNA response to epileptic preconditioning identifies miR-184 as a modulator of seizure-induced neuronal death. Exp Neurol. 2012;237(2):346-54.

46. Zhao C, Sun G, Li S, Lang MF, Yang S, Li W, Shi Y. MicroRNA let-7b regulates neural stem cell proliferation and differentiation by targeting nuclear receptor TLX signaling. Proc Natl Acad Sci U S A. 2010;107(5):1876-81.

47. Stappert L, Borghese L, Roese-Koerner B, Weinhold S, Koch P, Terstegge S, Uhrberg M, Wernet $P$, Brüstle O. MicroRNA-based promotion of human neuronal differentiation and subtype specification. PLoS One. 2013;8(3):e59011.

48. Agostini M, Tucci P, Killick R, Candi E, Sayan BS, di Val Cervo Rivetti P, Nicotera P, McKeon F, Knight RA, Mak TW, Melino G. Neuronal differentiation by TAp73 is mediated by microRNA-34a regulation of synaptic protein targets. Proc Natl Acad Sci U S A. 2011;108(52):21093-8.

49. Delaloy C, Liu L, Lee JA, Su H, Shen F, Yang GY, Young WL, Ivey KN, Gao FB. MicroRNA-9 coordinates proliferation and migration of human embryonic stem cell-derived neural progenitors. Cell Stem Cell. 2010;6(4):323-35.

50. Sun G, Ye P, Murai K, Lang MF, Li S, Zhang H, Li W, Fu C, Yin J, Wang A, Ma $X$, Shi Y. miR-137 forms a regulatory loop with nuclear receptor TLX and LSD1 in neural stem cells. Nat Commun. 2011;2:529.

51. Edbauer D, Neilson JR, Fos-ter KA, Wang CF, See-burg DP, Batterton MN, et al. Regulation of synaptic structure and function by FMRP- associated microRNAs miR-125b and miR-132. Neuron. 2010;65:373-84

52. Lukiw WJ, Zhao Y, Cui JG. An NF-kappaB-sensitive microRNA-146a-mediated inflammatory circuit in Alzheimer disease and in stressed human brain cells. J Biol Chem. 2008;283(46):31315-22.

53. Schaefer $A, O^{\prime}$ Carroll $D$, Tan $C L$, et al. Cerebellar neurodegeneration in the absence of microRNAs. J Exp Med. 2007;204(7):1553-8.

54. Davis TH, Cuellar TL, Koch SM, et al. Conditional loss of Dicer disrupts cellular and tissue morphogenesis in the cortex and hippocampus. J Neurosci. 2008;28:4322-30

55. Kaneko H, Dridi S, Tarallo V, et al. DICER1 deficit induces Alu RNA toxicity in age-related macular degeneration. Nature. 2011;471:325-30.

56. Tao J, Wu H, Lin Q, et al. Deletion of astroglial dicer causes non-cell-autonomous neuronal dysfunction and degeneration. J Neurosci. 2011;31:8306-19.

57. Hebert SS, Papadopoulou AS, Smith P, et al. Genetic ablation of Dicer in adult forebrain neurons results in abnormal tau hyperphosphorylation and neurodegeneration. Hum Mol Genet. 2010;19:3959-69.

58. Cifuentes $D$, Xue H, Taylor DW, Patnode H, Mishima Y, et al. A novel miRNA processing pathway independent of Dicer requires Argonaute2 catalytic activity. Science. 2010;328:1694-8.

59. Yang JS, Maurin T, Robine N, et al. Conserved vertebrate mir-451 provides a platform for Dicer independent, Ago2-meidated microRNA biogenesis. Proc Natl Acad Sci U S A. 2010;107(34):15163-8.

60. Nudelman AS, DiRocco DP, Lambert TJ, Garelick MG, Le J, et al. Neuronal activity rapidly induces transcription of the CREB-regulated microRNA-132, in vivo. Hippocampus. 2010;20:492-8.

61. Fiore R, Khudayberdiev S, Christensen M, Siegel G, Flavell SW, et al. Mef2mediated transcription of the miR379-410 cluster regulates activitydependent dendritogenesis by fine-tuning Pumilio2 protein levels. EMBO J. 2009;28:697-710

62. Wang X, Sun Y, Tan Z, Che N, Ji A, Luo X, Sun X, Li X, Yang K, Wang G, Luan L, Liu Y, Wei M, Yin J. Serum microRNA-4521 is a potential biomarker for focal cortical dysplasia with refractory epilepsy. Neurochem Res. 2016;41(4) 905-12.

63. Wang J, Tan L, Tan L, Tian Y, Ma J, Tan CC, Wang HF, Liu Y, Tan MS, Jiang T, Yu JT. Circulating microRNAs are promising novel biomarkers for drugresistant epilepsy. Sci Rep. 2015;5:10201.

64. Li MM, Li XM, Zheng XP, Yu JT, Tan L. MicroRNAs dysregulation in epilepsy. Brain Res. 2014;1584:94-104.

\section{Submit your next manuscript to BioMed Central and we will help you at every step:}

- We accept pre-submission inquiries

- Our selector tool helps you to find the most relevant journal

- We provide round the clock customer support

- Convenient online submission

- Thorough peer review

- Inclusion in PubMed and all major indexing services

- Maximum visibility for your research

Submit your manuscript at www.biomedcentral.com/submit
) Biomed Central 